\title{
Synthesis of the Silver Nanoparticle by Chemical Reduction Method and Preparation of Nanocomposite based on AgNPS
}

\author{
Hamed Ahari ${ }^{*}$, Guity Karim², Amir Ali Anvar³, Mobina Pooyamanesh ${ }^{4}$, Alireza Sajadi4, \\ Atefeh Mostaghim ${ }^{4}$, Sogand Heydari ${ }^{4}$ \\ ${ }^{1}$ Department of Food Science and Technology, Science and Research Branch, Islamic Azad University \\ Tehran, Iran \\ h.ahari@srbiau.ac.ir* corresponding author \\ ${ }^{2}$ Department of Food Hygiene, Faculty of Veterinary Medicine, University of Tehran \\ Tehran, Iran \\ ${ }^{3}$ Department of Hygiene, Science and Research Branch, Islamic Azad University \\ Tehran, Iran \\ ${ }^{4}$ Science and Research Branch, Islamic Azad University \\ Tehran, Iran
}

\begin{abstract}
Nanopackaging is one of the most significant subjects in food packaging And it is so important for food safety and food quality control, then we need natural method (without chemical material)for packaging process. In this study, the possibility of using nanocomposite (LDPE) based on silver nanoparticle as a food packaging was proposed. Chemical reduction method with Trisodium citrate as a reductant was applied for preparing silver nanoparticle. In the method mentioned using of diverse reducing agent can be produced different size of nanoparticles that having different antibacterial action. The nanostructure and particle size of of silvernanoparticles were confirmed by scanning electron microscopy(SEM). The average of particle size was $68 \mathrm{~nm}$. After confirming the formation of nanoparticles, they were coated on the LDPE surface by melt mixing method. Surface morphology of the silver/LDPE nanocomposite was characterised by Transmission Electron Microscopy (TEM), Energy Dispersive X-ray (EDX) and X-ray Photoelectron Spectroscopy (XPS). The film thickness was $33.67 \mathrm{~nm}$. In addition, the antibacterial properties of the prepared nanocomposite film was evaluated by disc diffusion method. The results showed avourable antibacterial efficiency against Escheria Coli (ATCC 8739) and staphylococcus aureus(ATCC 6538) and The results show that the released silver from the coated film is enough to create antimicrobial activity in the incubated solution.
\end{abstract}

Keywords: Polyethylene, Silver Nanocomposite, Chemical Reduction, Silver Nanoparticles, Antimicrobial Activity.

\section{Introduction}

Nanotechnology is emerging as a rapidly growing field with its application in science and technology for the purpose of manufacturing new materials at the nanoscale level [1]. Reduction of size to the nanometer scale increases surface-tovolume ratio and consequently the reactivity of nanoparticles[2]. A nanoparticle is a microscopic particle with size less than $100 \mathrm{~nm}$ [3]. The exceptional characteristics of nanosilver particles (AgNP) as biocide have made them the largest and fastest growing class of manufactured nanomaterials in commercial applications [4]. AgNP seem to work by damaging the structure of the bacterial cell membrane, creating pits and increasing its permeability, inhibiting respiratory enzymes, damaging bacterial DNA, and releasing Ag+ ions, thus causing the death of the exposed bacteria[5, 6]. The preparation of $\mathrm{Ag}$ nanoparticles usually involves such methods as vacuum evaporation, electrical arc discharge, microemulsion, phase transfer, and chemical reduction-protection. Among them, chemical reduction method is preferable because it has many merits such as mild reaction condition, high yield, low energy consumption, and simple separating proce- dure[7]. In one of the studies, a method was proposed for the fabrication of antibacterial nanocomposite polymeric films, for use in food packaging. a stable colloidal solution of silver nanoparticles was prepared by chemical reduction of silver saltusing fructose as a reducing agent. silver nanoparticles were coated on the LDPE surface by immersion of the treated films in the colloidal silver solutions. Surface morphology of the silver/LDPE nanocomposite was characterized by FE-SEM and AFM analysis[8]. It is possible to control the size and hence of optical properties of Ag nanoparticles simply by varying the reducing agent concentration[9]. A reducing agent is a chemical agent, plant extract, biological agent, or irradiation method that provides the free electrons needed to reduce silver ions and to form silver nanoparticles[10]. Ajitha et al synthesized silver nanoparticle by using trisodium citrate as reducing agent and 
demonstrated the effect of reductant concentration on size variation[11]. In other study, Silver nanoparticles were prepared by chemical reduction method and hydrazine hydrate was applied as a reducing agent.In the next step they were monitored by absorbtion spectroscopy. The average size of AgNPs were specified by transmission electron microscopy(TEM) as well[12]. Janardhanan et al presented A simple wet chemical route to synthesize nano-sized silver particles, and their surface properties are discussed in detail[9]. Effective antimicrobial nanocomposite packaging materials based on silver nanoparticles(AgNPs) have been presented. This was illustrated by Mayorga et al who produced silver nanoparticles (AgNPs) into a mixed microbial cultures based poly(3-hydroxybutyrate-co-18 mol\%- 3hydroxyvalerate)(PHBV18) was used, which was diluted by melt compounding with a commercial poly(3hydroxybutyrate-co-3 mol\%- 3-hydroxyvalerate) (PHBV3) material[13]. In the study that presented by Shrivastava et al silver nanoparticles were prepared in the range of $10-15 \mathrm{~nm}$ and the morphology of the nanoparticles was characterized by transmission electron microscopy[14]. In another study, Colloidal silver nanoparticles with a size of $5.5 \pm 1.1 \mathrm{~nm}$ were prepared by chemical reduction using polyethylene glycol (PEG). low-density polyethylene (LDPE) synthesized by melt blending and subsequent hot pressing to produce nanocomposite film. PEG was added at 5\% weight of polymer as a compatibilizer agent in order to prevent agglomeration and provide uniform distribution of nanoparticles in polymer matrix. Antimicrobial activity of silver nanocomposites against pathogenic bacteria was evaluated by semi-qualitative agar diffusion test and quantitative dynamic shake flask test[15]. Morones et al tested silver nanoparticles in four types of Gram-negative bacteria: E. coli, V. cholera, P. aeruginosa and S. typhus[5]. The antibacterial activity and acting mechanism of silver nanoparticles (SNPs) on Escherichia coli ATCC 8739 were investigated by Li et al,who analyzed the growth, permeability, and morphology of the bacterial cells following treatment with SNPs [6]. Activity of silver nanoparticles against E. coli was investigated by Sondi et al. Bacteriological tests were performed in Luria-Bertani (LB) medium on solid agar plates and in liquid systems supplemented with different concentrations of nanosized silver particles[16]. In other study Park et al evaluated the antimicrobial activity of silver nanoparticles which were prepared using different stabilizer, such as sodium dodecylsulfate (SDS) and poly-(N-vinyl-2-pyrrolidone) (PVP), for Staphylococcus aureus (S. aureus) and Escherichia coli (E.coli) by measuring the minimum inhibitory concentration[17]. Antibacterial properties of AgNPs was improved by increasing percentage of concentration or decreasing the size of AgNPs, however the migration rate of nanoparticle was increased significantly. Simon et al investigated the effect of nanoparticle size on migration from polymer packaging to food[18]. In other study Song et al evaluated the migration of Ag from nanosilver- polyethylene composite packaging into food simulants at different temperatures and times[19]. The aim of this study is to fabricate silver/polyethylene nanocomposite films for use as antimicrobial packaging. For this purpose, a colloidal silver nanoparticle solution was prepared by reduction of silver salt using Trisodium Citrate. silver nanoparticles were coated on the surface of treaded LDPE films. The silver nanocomposite films were tested by FE-SEM and AFM analysis and the quantity of coated silver on the films as well as silver ion release from the fabricated films was measured.Furthermore, the antibacterial properties of nanocomposite films were determined.

\section{Materials and Methods}

\subsection{Synthesis of Silver NPs}

First of all, $0.0849 \mathrm{gr} \mathrm{AgNO} 3$ was dissolved in $500 \mathrm{ml}$ distilled water,then $5 \mathrm{gr}$ of Trisodium Citrate solution was added to the $100 \mathrm{ml}$ boiling AgNO3.The solution was placed at $90^{\circ} \mathrm{C}$ for 2 hours. Finally the solution's color changed to red.

\subsection{Preparation of Antimicrobial Nanocomposite Films}

Nanocomposite film (LDPE) were made by melt-mixing method. Along the screw were various screw elements in order to instigate polymer melting and to achieve suitable dispersion of the nanoparticles in the polymer melt[20]. fine dispersion of the minor component in the polymer matrix was observed for all the studied blends with scanning electron microscopy[21].

\subsection{Characterization of the AgNPS}

Electron microscopy imaging was used in order to determine the characteristics of nanoparticles and investigate the size of them, Suspensions were made in solvent within laboratory flasks to prepare samples of electron microscopy. $3 \mathrm{ml}$ of the solution was placed to evaporate the solvent. Samples coated by gold and transferred to the Spotter coater device. After 10 minutes the samples were transferred to the electron microscope case,the electron bombardment was applied and the images were recorded[4].

\subsection{Scanning Electron Microscopy - Energy Dispersive X-Ray}


Scanning electron microscopy - energy dispersive x-ray Silver NPs present in the coated films were identified and characterised using scanning electron microscopy equipped with energy dispersive $\mathrm{x}$-ray[22].

\subsection{Determination of AgNP Loading in the Coatings}

The total level of $\mathrm{Ag}$ found in each of the coatings $(\mathrm{n}=3)$ was determined by total acid digestion of the LDPE and PT films.Films $(2.5 \mathrm{~cm} \times 2.5 \mathrm{~cm})$ were cut into $1 \mathrm{~mm} \times 1 \mathrm{~mm}$ squares and placed in a PTFE vessel containing $10 \mathrm{ml} 69 \%$ HNO3 (69\% HNO3, VWR International, Ireland). The samples were then incubated at $120{ }^{\circ} \mathrm{C}$ for $5 \mathrm{~h}$ in an oven. Following total digestion, the acid solutions containing the digested films in the absence of any residual LDPE or PT were sampled and $100 \mu \mathrm{l}$ of the digestate was diluted with $9.9 \mathrm{ml}$ Milli-Q water (18.2M $\Omega \mathrm{cm}$, PURELAB Option-Q, Elga, U.K.) and analysed immediately using ICPAES[22].

\subsection{Antibacterial Test}

The bacterial strains used in this study were Staphylococcus aureus (ATCC 6538), Escherichia coli (ATCC 8739) which were provided by the iranian research organization for science and technology.Manitol salt agar media and eosin methylene blue media were used as specific media for S. aureus and E. coli, respectively and PDA,TSA media was used to examine the antimicrobial properties of the cultures. Each film sample of $1 \times 1 \mathrm{~cm}^{2}$ in size was placed on the medium. After incubation at $37^{\circ} \mathrm{c}$ for $24 \mathrm{~h}$ inhibition of zone were measured[8].

\section{Results and Discussion}

\subsection{Optical Absorption Test in the Infrared Spectral Region}

The purpose of this test is to qualitative and quantitative evaluation of the organic compounds contain nanoparticle, Identify the type of functional groups and chemical bonds in the coating molecules. For this purpose, the film sample was cut of $10 \mathrm{~cm}$ in size and placed on the sample location and characterized by dispersive X-ray. Two groups of films were examined (freezer bag as a control group and $\mathrm{Ag}$ nanocomposite film).

\subsection{FE-SEM Analysis}

In order to measure nanoparticles and particle dispersion FE-SEM and FTIR were used to determine the type of chemical bond.as seen in figures below, the distribution of silver nanoparticle with $20 \mathrm{KV}, 30$ and $60 \mathrm{KX}$ is measured. The average of particle size is $68 \mathrm{~nm}$. Also the film thickness was $33.67 \mathrm{~nm}$. Consistancy of the Ag coated film was evident by FE-SEM electron microscope. At points that investigated with more magnification, presence of accumulation was obvious which was due to the lack of a perfect solution within the sample coatings using the acetonitrile solvent.

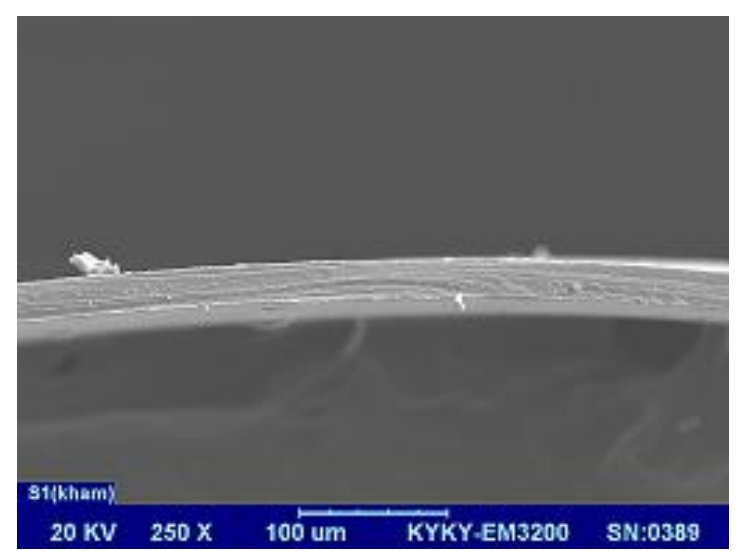

Fig. 1: The SEM image from the surface of the Ag /nanocomposite. 


\subsection{Antibacterial activity of silver/ nanocomposite}
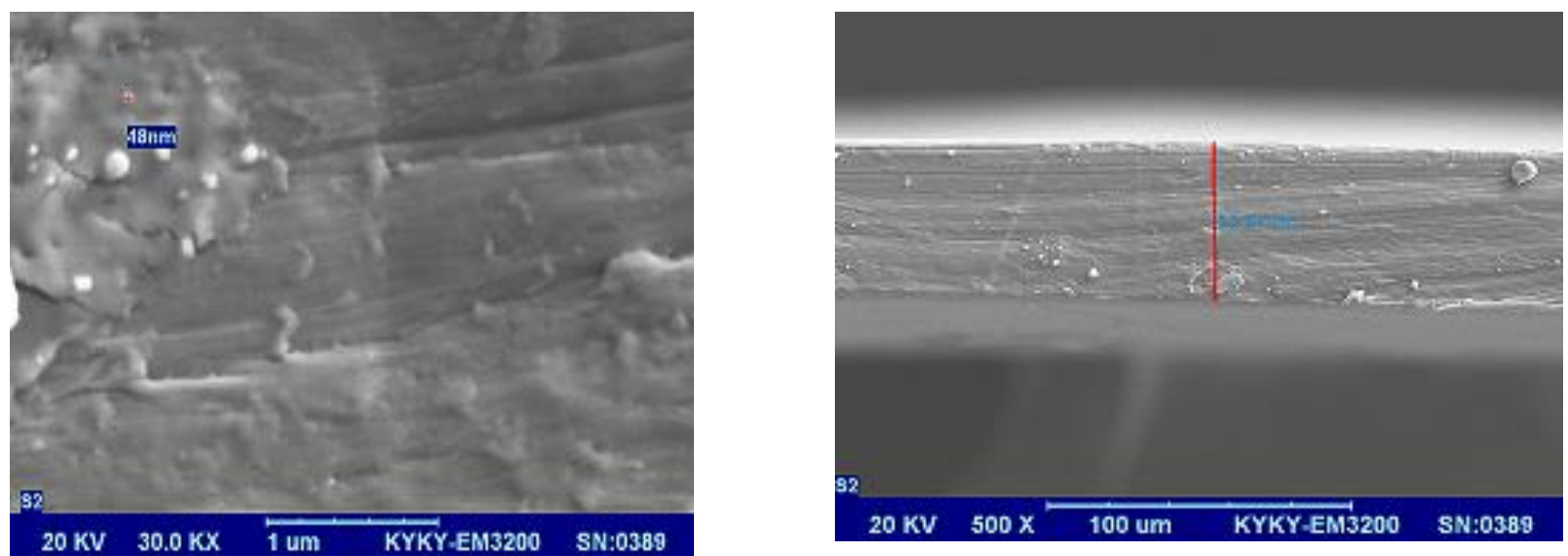

coating of Silver nanoparticle on the LDPE films exposed an interesting antibacterial activity in the agar disc diffusion test. The antimicrobial activity of silver nanoparticles synthesized by natural plants extract was investigated against pathogenic organisms such as S. aureus, E. coli Colonies of bacteria could not be seen in the clear zone directly around the silver coated films. The results show that the released silver from the coated film is enough to create antimicrobial activity in the incubated solution.

\section{Discussion}

In this study chemical reduction method was used to synthesis silver nanoparticle. In order to confirm the efficiency of this method, Dehnavi et al applied this approach by using corona air plasma as a pretreatment of LDPE films to increase the adhesion of silver nanoparticle on the film surface. Jo et al asserted that the Ag/LDPE nanocomposite film has a comparable strength to that of commercial LDPE, although stiffness was increased at high Ag (240 mg/kg) concentration[23]. It is notable that using of diverse reducing agent can be produced different size of nanoparticle that having different antibacterial action. In the present study, Trisodium Citrate was used as a reduce agent. In this regard sileikaite et al reported that the synthesized of silver nano particles from AgNO3 salt were 100 nm when sodium citrate was used as reducing agent[24]. The results of scanning electron microscopy implied the size of $68 \mathrm{~nm}$ as the average value of silver nanoparticles.In one of the studies the FESEM images indicated that the average size of silver nanoparticles reduced from $30 \mathrm{~nm}$ to $18 \mathrm{~nm}$ on addition of $2 \mathrm{M}$ reducing agent[11]. The averege tickness of Silver Nanocomposite was $33.67 \mathrm{~nm}$. In line with the results obtained Jabra and Ismail stated the size of $40 \mathrm{~nm}$ as the mean value by using glucose as a reductant[25]. In other study that reported by Jokar et al silver nanoparticle were prepared by chemical reduction using polyethylene glycol. the average size of AgNPs and tickness of nanocomposite film were 5.5 and $0.7 \mathrm{~nm}$ respectively[15]. Also Janardhanan et al confirmed that by increasing the concentration of the ethylene amine as reduce agent from 77 to $115 \mathrm{mM}$, the size of nanoparticles reduced. The images obtained from the electron microscope showed that the average particle size of nanoparticle decreased from 75 to 45 nm[9]. In our study FE-SEM images with more magnification showed the presence of accumulation due to the lack of perfect solution. In line with the result obtained, Jo et al evaluated the morphology of the nanocomposite films and the dispersion of the AgNP by FESEM images and observed the white spots represent an agglomeration of AgNP as confirmed by the results of EDS[23]. As expected, the effects of Silver Nanocomposite on both gram positive and negative bacteria is quite obvious. By increasing the percentages of nanosilver, microbial load decreased. The antimicrobial activity of these nanoparticles may be related to several mechanisms. Sawai \& Yoshikawa concluded that induction of oxidative stress due to generation of reactive oxygen species (ROS) may cause the degradation of the membrane structure of the cell[26], In accordance with this results, Dill et al showed silver nanocomposite could effect on pathogenic bacteria[12]. Also Mayorga et al illustrated that PHBvs-AgNPs film has a strong antibacterial activity[13]. In other study,Morris et al represented that nanocomposite films containing AgNPs could potentially be used as antimicrobial packaging for food applications [27]. moreover in recent study Tavakoli et al demonstrated that using of different concentration of nano silver 
could enhance the shelf life of the nuts by decreasing of microbial load[28]. In contrast, Jokar et al revealed antibacterial impact of silver nanocomposite on gram positive bacteria (S.aureus) was more efficient than gram negative (E.coli)[15]. Also Shrivastava et al noted that silver nanoparticles in the range of 10-15 $\mathrm{nm}$ have a higher antibacterial impact on gram negative than gram-positive bacteria[14]. Morones et al. reported that silver nanoparticles of 1-10 nm must be compact and connected to each other to have the highest antimicrobial effects[5]. Park et al evaluated the antimicrobial activity of silver nanoparticles for Staphylococcus aureus (S. aureus) and Escherichia coli (E.coli) by measuring the minimum, the MIC of Ag-NPs for S. aureus and E. coli were 5 and 10 ppm, respectively[17]. In othr study activity of silver nanoparticles against $\mathbf{E}$. coli was investigated by Sondi et al. Bacteriological tests were performed in Luria-Bertani (LB) medium on solid agar plates and in liquid systems supplemented with different concentrations of nanosized silver particles.The results confirmed that the treated E. coli cells were damaged, showing formation of "pits" in the cell wall of the bacteria, while the silver nanoparticles were found to accumulate in the bacterial membrane[16]. Mirzajani et al. clarified the mechanism of the effect of silver nanoparticles on $S$. aureus These authors noted that by adding silver nanoparticles at $20 \mathrm{~nm}$ to the $S$. aureus culture, the amount of muramic acid released in the environment increased[29]. Li et al observed that using of $10 \mu \mathrm{g} / \mathrm{ml}$ SNPs had significant fatal effect onE.coli bacteria[6].ŠIMON et al represented that any detectable migration of The ENP from packaging to food will take place in the case of very small ENPs with a radius of $1 \mathrm{~nm}[18]$. 
Proceedings of the $4^{\text {th }}$ World Congress on Mechanical, Chemical, and Material Engineering (MCM'18)

Madrid, Spain-August 16-18, 2018

Paper No. ICCPE XXX (The number assigned by the OpenConf System)

DOI: TBA

\section{References}

[1] P. Logeswari, S. Silambarasan, and J. Abraham, "Synthesis of silver nanoparticles using plants extract and analysis of their antimicrobial property," Journal of Saudi Chemical Society, vol. 19, no. 3, pp. 311-317, 2015.

[2] P. Ezhilarasi, P. Karthik, N. Chhanwal, and C. Anandharamakrishnan, "Nanoencapsulation techniques for food bioactive components: a review," Food and bioprocess technology, vol. 6, no. 3, pp. 628-647, 2013.

[3] S. M. Roopan et al., "Efficient phyto-synthesis and structural characterization of rutile TiO2 nanoparticles using Annona squamosa peel extract," Spectrochimica Acta Part A: Molecular and Biomolecular Spectroscopy, vol. 98, pp. 86-90, 2012.

[4] N. C. Martins et al., "Electrostatic assembly of Ag nanoparticles onto nanofibrillated cellulose for antibacterial paper products," Cellulose, vol. 19, no. 4, pp. 1425-1436, 2012.

[5] J. R. Morones et al., "The bactericidal effect of silver nanoparticles," Nanotechnology, vol. 16, no. 10, p. $2346,2005$.

[6] W.-R. Li, X.-B. Xie, Q.-S. Shi, H.-Y. Zeng, O.-Y. You-Sheng, and Y.-B. Chen, "Antibacterial activity and mechanism of silver nanoparticles on Escherichia coli," Appl Microbiol Biotechnol, vol. 85, no. 4, pp. 1115-1122, 2010.

[7] J. Liu, X. Li, and X. Zeng, "Silver nanoparticles prepared by chemical reduction-protection method, and their application in electrically conductive silver nanopaste," Journal of Alloys and Compounds, vol. 494, no. 1-2, pp. 8487, 2010.

[8] A. S. Dehnavi, A. Aroujalian, A. Raisi, and S. Fazel, "Preparation and characterization of polyethylene/silver nanocomposite films with antibacterial activity," Journal of Applied Polymer Science, vol. 127, no. 2, pp. 1180-1190, 2013.

[9] R. Janardhanan, M. Karuppaiah, N. Hebalkar, and T. N. Rao, "Synthesis and surface chemistry of nano silver particles," Polyhedron, vol. 28, no. 12, pp. 2522-2530, 2009.

[10] T. M. Tolaymat, A. M. El Badawy, A. Genaidy, K. G. Scheckel, T. P. Luxton, and M. Suidan, "An evidence-based environmental perspective of manufactured silver nanoparticle in syntheses and applications: a systematic review and critical appraisal of peer-reviewed scientific papers," Science of the Total Environment, vol. 408, no. 5, pp. 999-1006, 2010.

[11] B. Ajitha, A. Divya, K. S. Kumar, and P. S. Reddy, "Synthesis of silver nanoparticles by soft chemical method: Effect of reducing agent concentration," in Advanced Nanomaterials and Emerging Engineering Technologies (ICANMEET), 2013 International Conference on, 2013, pp. 7-10: IEEE.

[12] M. G. Guzmán, J. Dille, and S. Godet, "Synthesis of silver nanoparticles by chemical reduction method and their antibacterial activity," Int J Chem Biomol Eng, vol. 2, no. 3, pp. 104-111, 2009.

[13] J. Castro-Mayorga, M. Fabra, and J. Lagaron, "Stabilized nanosilver based antimicrobial poly (3-hydroxybutyrate-co3-hydroxyvalerate) nanocomposites of interest in active food packaging," Innovative Food Science \& Emerging Technologies, vol. 33, pp. 524-533, 2016.

[14] S. Shrivastava, T. Bera, A. Roy, G. Singh, P. Ramachandrarao, and D. Dash, "Characterization of enhanced antibacterial effects of novel silver nanoparticles," Nanotechnology, vol. 18, no. 22, p. 225103, 2007.

[15] M. Jokar, R. A. Rahman, N. A. Ibrahim, L. C. Abdullah, and C. P. Tan, "Melt production and antimicrobial efficiency of low-density polyethylene (LDPE)-silver nanocomposite film," Food and bioprocess technology, vol. 5, no. 2, pp. 719-728, 2012.

[16] I. Sondi and B. Salopek-Sondi, "Silver nanoparticles as antimicrobial agent: a case study on E. coli as a model for Gram-negative bacteria," J Colloid Interface Sci, vol. 275, no. 1, pp. 177-182, 2004.

[17] K.-H. Cho, J.-E. Park, T. Osaka, and S.-G. Park, "The study of antimicrobial activity and preservative effects of nanosilver ingredient," Electrochimica Acta, vol. 51, no. 5, pp. 956-960, 2005.

[18] P. ŠIMON, Q. Chaudhry, and D. BAKOŠ, "Migration of engineered nanoparticles from polymer packaging to food-a physicochemical view," Journal of Food \& Nutrition Research, vol. 47, no. 3, 2008.

[19] H. Song, B. Li, Q.-B. Lin, H.-J. Wu, and Y. Chen, "Migration of silver from nanosilver-polyethylene composite packaging into food simulants," Food Additives \& Contaminants: Part A, vol. 28, no. 12, pp. 1758-1762, 2011.

[20] D. N. Bikiaris, G. Z. Papageorgiou, E. Pavlidou, N. Vouroutzis, P. Palatzoglou, and G. P. Karayannidis, "Preparation by melt mixing and characterization of isotactic polypropylene/SiO2 nanocomposites containing untreated and surface-treated nanoparticles," Journal of Applied Polymer Science, vol. 100, no. 4, pp. 2684-2696, 2006. 
[21] G. Takidis, D. Bikiaris, G. Papageorgiou, D. Achilias, and I. Sideridou, "Compatibility of low-density polyethylene/poly (ethylene-co-vinyl acetate) binary blends prepared by melt mixing," Journal of Applied Polymer Science, vol. 90, no. 3, pp. 841-852, 2003.

[22] J. C. Hannon, J. P. Kerry, M. Cruz-Romero, S. Azlin-Hasim, M. Morris, and E. Cummins, "Migration assessment of silver from nanosilver spray coated low density polyethylene or polyester films into milk," Food Packaging and Shelf Life, vol. 15, pp. 144-150, 2018.

[23] Y. Jo et al., "Characterization and antibacterial properties of nanosilver-applied polyethylene and polypropylene composite films for food packaging applications," Food Bioscience, vol. 23, pp. 83-90, 2018.

[24] A. Šileikaitè, I. Prosyčevas, J. Puišo, A. Juraitis, and A. Guobienè, "Analysis of silver nanoparticles produced by chemical reduction of silver salt solution," Mater. Sci, vol. 12, no. 4, pp. 1392-1320, 2006.

[25] M. Ismail and R. Jabra, "Investigation the parameters affecting on the synthesis of silver nanoparticles by chemical reduction method and printing a conductive pattern," 2017.

[26] J. Sawai and T. Yoshikawa, "Quantitative evaluation of antifungal activity of metallic oxide powders $(\mathrm{MgO}, \mathrm{CaO}$ and $\mathrm{ZnO}$ ) by an indirect conductimetric assay," J Appl Microbiol, vol. 96, no. 4, pp. 803-809, 2004.

[27] S. Azlin-Hasim, M. C. Cruz-Romero, M. A. Morris, E. Cummins, and J. P. Kerry, "Effects of a combination of antimicrobial silver low density polyethylene nanocomposite films and modified atmosphere packaging on the shelf life of chicken breast fillets," Food Packaging and Shelf Life, vol. 4, pp. 26-35, 2015.

[28] H. Tavakoli, H. Rastegar, M. Taherian, M. Samadi, and H. Rostami, "The effect of nanosilver packaging in increasing the shelf life of nuts: An in vitro model," Ital J Food Saf, vol. 6, no. 4, 2017.

[29] F. Mirzajani, A. Ghassempour, A. Aliahmadi, and M. A. Esmaeili, "Antibacterial effect of silver nanoparticles on Staphylococcus aureus," Research in microbiology, vol. 162, no. 5, pp. 542-549, 2011. 